\title{
DIMENSÃO FRACTAL DE SÉRIES TEMPORAIS MEDIDAS ACIMA DO DOSSEL DE FLORESTA NO PANTANAL MATO-GROSSENSE
}

MELLO, Geison Jader - geison.mello@svc.ifmt.edu.br

Instituto Federal de Mato Grosso (IFMT)

Prog. de Pós-Graduação em Física Ambiental (PGFA), Univ. Fed. de Mato Grosso (UFMT)

PAULO, Iramaia Jorge Cabral de - ira@cpd.ufmt.br

Prog. de Pós-Graduação em Física Ambiental (PGFA), Univ. Fed. de Mato Grosso (UFMT)

PAULO, Sergio Roberto de - sergio@ufmt.br

Prog. de Pós-Graduação em Física Ambiental (PGFA), Univ. Fed. de Mato Grosso (UFMT)

GOMES, Raphael de Souza Rosa - thesivis@gmail.com

Prog. de Pós-Graduação em Física Ambiental (PGFA), Univ. Fed. de Mato Grosso (UFMT)

MACHADO, Nadja Gomes - nadja.machado@blv.ifmt.edu.br

Instituto Federal de Mato Grosso (IFMT)

Prog. de Pós-Graduação em Física Ambiental (PGFA), Univ. Fed. de Mato Grosso (UFMT)

NOGUEIRA, José de Souza - nogueira@ufmt.br

Prog. de Pós-Graduação em Física Ambiental (PGFA), Univ. Fed. de Mato Grosso (UFMT)

BIUDES, Marcelo Sacardi - marcelo.biudes@gmail.com

Prog. de Pós-Graduação em Física Ambiental (PGFA), Univ. Fed. de Mato Grosso (UFMT)

Resumo: Analisamos dados medidos acima da copa da floresta em torre micrometeorológica no Pantanal Brasileiro procurando por atratores caóticos de baixa dimensionalidade nas variáveis balanço de radiação $(R n)$, temperatura $(T)$ e umidade relativa do ar (UR) no período de 2007 a 2009. As estimativas da dimensão de correlação $\left(D_{2(R n)}=1,60 \pm 0,05 ; D_{2(T)}=2,08 \pm 0,08 ; D_{2(U R)}=2,16 \pm 0,07\right)$ uma aproximação da dimensão fractal e a dimensão de imersão $\left(n_{(R n)}=7 ; n_{(T)}=8 ; n_{(U R)}=9\right)$ sugerem existência de atratores caóticos de baixa dimensão com sensível variação sazonal, sendo utilizado para os cálculos o algoritmo de Grassberger e Procaccia.

Palavras-chave: dimensão de correlação; atratores; sazonalidade; floresta tropical.

FRACTAL DIMENSION OF TIME SERIES OF MEASURES UP CANOPY IN FOREST AT PANTANAL OF MATO GROSSO

Abstract: We analyzed measured data above the forest canopy in a micrometeorological tower in the Brazilian Pantanal searching for low-dimensional chaotic attractors in the variables net radiation $(\mathrm{Rn})$, temperature $(\mathrm{T})$ and relative humidity $(\mathrm{RH})$ between 2007 to 2009. Estimates of dimension correlation, the fractal dimension $\left(D_{2(R n)}=1.60 \pm 0.05\right.$; $\left.D_{2(T)}=2.08 \pm 0.08 ; D_{2(U R)}=2.16 \pm 0.07\right)$ an approximation of the fractal dimension and the embedded dimension $\left(\mathrm{n}_{(\mathrm{Rn})}=7 ; \mathrm{n}_{(\mathrm{T})}=8 ; \mathrm{n}_{(\mathrm{UR})}=9\right)$ suggest the existence of low-dimensional chaotic attractors with significant seasonal variation, being used for the calculations the Grassberger and Procaccia algorithm.

Keywords: correlation dimension; attractors; seasonality; tropical forest. 


\section{INTRODUÇÃO}

O bioma Pantanal é uma das maiores planícies do planeta, com $250 \mathrm{mil} \mathrm{km}^{2}$ de extensão localizada entre os biomas Amazônico e Cerrado. Desempenha papel importante na estabilidade dinâmica do clima regional e global. A característica de maior distinção é a inundação sazonal, configuradora de clima, fauna e flora peculiares (JUNK \& NUNES DA CUNHA, 2005).

O aumento do desmatamento para a criação de gado pode modificar o clima local, levando a sérias consequências ecológicas, como o aumento da temperatura do ar, redução da precipitação e o prolongamento da estação seca (NOBRE et al., 1996; COSTA \& PIRES, 2010; LEWIS et al., 2011).

Destaca-se a importância da aplicação de ferramentas adequadas para se compreender os processos de troca de energia, matéria e momentum em floretas tropicais. Pesquisas recentes tem abordado a natureza caótica dos dados coletados em florestas tropicais contribuindo para o entendimento e modelagem da interação biosfera-atmosfera (CAMPANHARO et al., 2008). A partir da abordagem por teoria dos sistemas dinâmicos (NICOLIS \& PRIGOGINE, 1998; LORENZ, 1991; WALDROP, 1992; PEITGEN et al., 2004), várias pesquisas com séries temporais mencionam a possível existência de atratores climáticos de baixa dimensão caótica e indicam o número mínimo de variáveis presentes na evolução do sistema (RUELLE \& TAKENS, 1971; NICOLIS \& NICOLIS, 1984; FRAEDRICH，1986; POVEDO-JARAMILLO \& PUENTE，1993; XIN et al., 2001; GALLEGO et al., 2001).

Neste trabalho buscou-se detectar e analisar os atratores associados as séries temporais de balanço de radiação $(R n)$, temperatura do $\operatorname{ar}(T)$ e umidade relativa do ar (UR) do sistema dinâmico florestal do Pantanal Mato-Grossense, determinando sua dimensão fractal (dimensão de correlação $D_{2}$ ), a caoticidade da série temporal, e a dimensão de imersão $n$, usando 0 algoritmo de Grassberger \& Procaccia (1983).

\section{MATERIAL E MÉTODOS}

\subsection{Localização da Área Experimental e Instrumentação Utilizada}

A área experimental localiza-se na Reserva Particular do Patrimônio Natural RPPN SESC - Pantanal, município de Barão de Melgaço - MT, distante $160 \mathrm{~km}$ de Cuiabá, Mato Grosso, na Região Centro-Oeste do Brasil onde está instalada uma torre micrometeorológica de $32 \mathrm{~m}$ de altura (16039'50"S; 56047'50"O, altitude de $120 \mathrm{~m}$ ). A torre é contornada por uma vegetação florestal monodominante de cambará (Vochysia divergens), vulgo cambarazal, em uma faixa contínua de $25 \mathrm{~km}$ de extensão e $4 \mathrm{~km}$ de largura paralela ao rio Cuiabá (JUNK, 2002) com altura do dossel variando entre 28 a $30 \mathrm{~m}$. O cambarazal tem apresentado rápido espalhamento em campos sazonalmente inundados, efeito este atribuído às possíveis mudanças climáticas (ARIEIRA \& NUNES DA CUNHA, 2006). O solo é GLEISSOLO HÁPLICO Ta Distrófico (BELLAVER, 2010). O clima é 
AW segundo Köppen, a temperatura do ar média anual oscila entre $22^{\circ} \mathrm{C}$ e $32^{\circ} \mathrm{C}$ e a precipitação média anual entre 1100 e 1200 mm (HASENACK et al., 2003) com período seco de maio a setembro (CAMPELO JUNIOR et al., 1991).

Foram analisados os dados de saldo de radiação $(R n)$, temperatura do ar $(T)$ e umidade relativa do ar (UR) devido ao forte acoplamento dessas variáveis no espaço de fase dos sistemas climáticos (PEREIRA et al., 2002). As medidas de $\mathrm{Rn}$ foram feitas com um saldo radiômetro (Net Radiometer, Kipp \& Zonen Delft, Inc., Holland) instalado a $32 \mathrm{~m}$ de altura. As medidas de T e UR foram feitas utilizando um termohigrômetro (HMP 45 C, Vaisala, Inc., Helsinki, Finland) instalado em a uma haste a $40 \mathrm{~m}$ de altura (10 $\mathrm{m}$ acima do dossel da floresta). Esses dados foram coletados a $0,2 \mathrm{~Hz}$ e armazenados com médias de 15 minutos em datalogger (CR 10X, Campbell Scientific, Inc., Ogden, Utah) perfazendo aproximadamente 3000 dados mensais para cada atributo climático. Além do período seco e chuvoso também foi analisado o período alagado, de março a maio, característica peculiar do Pantanal. Os dados deste trabalho são referentes ao período de julho de 2007 à maio de 2009, perfazendo um espectro de dois anos.

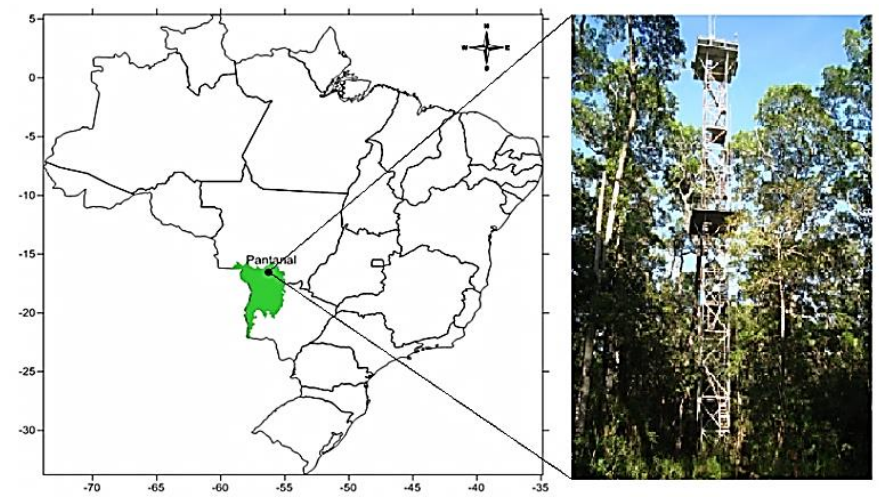

Figura 1. Mapa do Brasil, com localização da área de vegetação monodominante de Cambará na RPPN SESC - Pantanal (Cambarazal) e a torre micrometeorológica.

\begin{tabular}{lc}
\hline \multicolumn{1}{c}{ Ano / Meses } & Sazonalidade \\
\hline 2007 / julho e agosto & Período seco \\
2008 / janeiro e fevereiro & Período chuvoso \\
2008 / março, abril e maio & Período inundado \\
2008 / julho e agosto & Período seco \\
2009 / janeiro e fevereiro & Período chuvoso \\
2009 / março, abril e maio & Período inundado \\
\hline
\end{tabular}

Tabela 1. Subdivisão dos dados por características sazonais.

\subsection{Método da Reconstrução do espaço de fase das variáveis}


As séries temporais (Figura $2 \mathrm{~A}$ ), entendida como uma sequência de observações no tempo, podem fornecer informações valiosas sobre a dinâmica subjacente da qual são provenientes.

A.

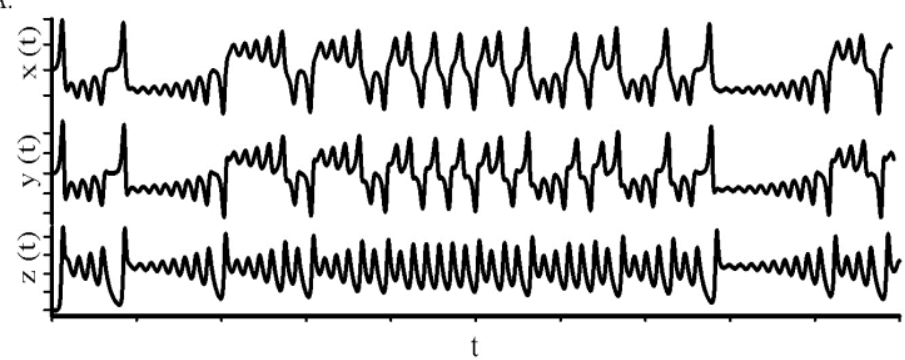

B.

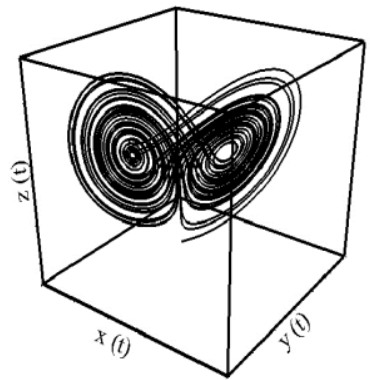

Figura 2. (A) solução numérica do sistema de equações de Lorenz (1963) para $x(t), y(t)$ e $z(t)$ e (B) representação do espaço de fase tridimensional do atrator estranho de Lorenz (parâmetros de controle $\rho=25, \sigma=10, \beta=8 / 3$ ).

Admitindo-se que uma série temporal é obtida de um sistema dinâmico com um número $\mathrm{m}$ de variáveis desconhecidas, é possível extrair mais informações dela do que apenas o padrão de comportamento da serie em si, visto nela conter as impressões das outras variáveis ocultas do sistema e que são acopladas ao seu espaço de fase $\mathrm{m}$ dimensional (Figura 2B). Usando as series de dados disponíveis empregamos algumas técnicas da teoria dos sistemas dinâmicos para desdobrar a estrutura $\mathrm{m}$ dimensional do espaço de fase das variáveis e sondar a estrutura dos atratores do sistema, ou seja, regiões específicas no espaço de fase para onde as trajetórias do sistema dinâmico convergem (LORENZ, 1963; NICOLIS \& PRIGOGINE, 1998). Este objeto geométrico de dimensão não inteira e estrutura auto similar que ser repete estatisticamente em todas as escalas de magnitude (fractal) é geralmente chamado de atrator estranho (RUELLE \& TAKENS, 1971)

\subsection{Método das defasagens temporais}

Umas das mais importantes técnicas de reconstrução do espaço de fase para identificar o atrator a partir de uma única série temporal é o método das defasagens temporais proposto por Floris Takens (1981). O espaço reconstruído apresenta uma suave variação de coordenada em relação ao espaço original, preservando os invariantes geométricos do sistema, como a dimensão fractal do atrator por exemplo (Figura $3 \mathrm{C}$ ). Este poderoso método utiliza apenas uma série temporal do sistema dinâmico, $\mathrm{X}_{0}(\mathrm{t})$, na qual o atrator está oculto para obter (ou reconstruir) as outras variáveis $\left\{X_{k}(t)\right\}$ (sendo $k=1, \ldots, n-1$ ) conhecendo somente $X_{0}(t)$. Desmembra-se a série temporal original $X_{0}(t)$ em deslocamentos temporais sucessivos de defasagem fixa $\tau(\operatorname{com} \tau=m \Delta t$, onde $m$ é um número inteiro e $\Delta t$ é um intervalo entre sucessivas amostras) para " $n$ " pontos equidistantes do conjunto de dados. Isto é: 


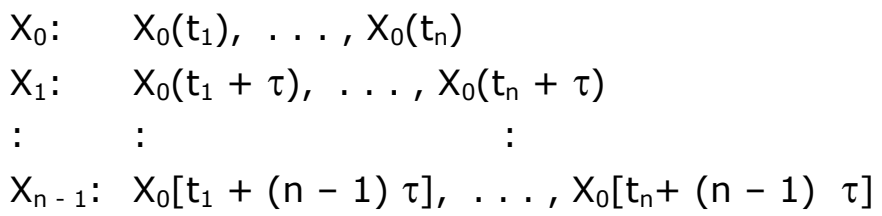

A melhor escolha de $\tau$ deve apresentar uma independência linear entre a série original e as séries defasadas, ou seja, a menor autocorrelação, no intuito de obter a maior informação possível do sistema estudado. A reconstrução do atrator com coordenadas de atraso temporal não é um processo automático, logo é importante a escolha de um tao $(\tau)$ apropriado (Figura 3 ) com afirma Baker \& Gollub (1996).
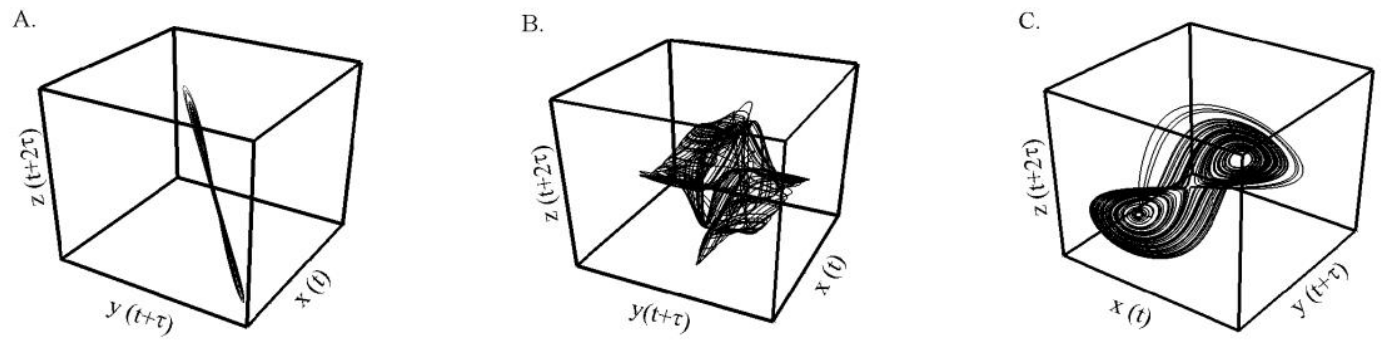

Figura 3. Efeito da defasagem temporal $\tau$ na reconstrução do atrator de Lorenz (1963) a partir da variável $x$ para $(A)$ uma defasagem $\tau$ muito pequena $(\tau=2)$, (B) uma defasagem $\tau$ muito grande $(\tau=50)$ e para (C) uma defasagem adequada $(\tau=10)$.

\subsection{Determinação do tao $(\tau)$ pela função autocorrelação acf $(\tau)$}

Em geral, os dados vizinhos em uma serie temporal são correlacionados, e para extrair o máximo de informação desta série utiliza-se a função autocorrelação $\operatorname{acf}(\tau)$, que estima o grau de correlação de uma variável em um dado instante consigo mesma a medida que o tempo passa, ou seja, a dependência entre o mesmo sinal defasado. O tempo de defasagem tao $(\tau)$ adequado é encontrado quando a função autocorrelação $\operatorname{acf}(\tau)$ atinge o valor zero ou o primeiro mínimo, característica da interdependência entre $X(t)$ e $X(t+\tau)$ :

$\operatorname{acf}(\tau)=\frac{1}{n-\tau} \sum_{t=1}^{n-\tau} \frac{\left(X_{(t)}-\bar{X}\right)\left(X_{(t+\tau)}-\bar{X}\right)}{\sigma^{2}}$

Onde $n$ é o numero de dados da série, $\bar{X}$ sua média e $\sigma^{2}$ sua variância. Plota-se $\operatorname{acf}(\tau)$ versus $\tau$ para visualizar a forma como a dependência da série cai com $0 \tau$ entre pontos da série. Para séries periódicas e quasiperiódicas a $\operatorname{acf}(\tau)$ permanece diferente de zero e igualmente periódica quando o tempo tende ao infinito, pois o sinal volta a parecer consigo após um período (Figura 4A); e para sistemas altamente não lineares $\operatorname{acf}(\tau)$ tende a zero quando $\tau$ tende ao infinito (Figura 4B). 


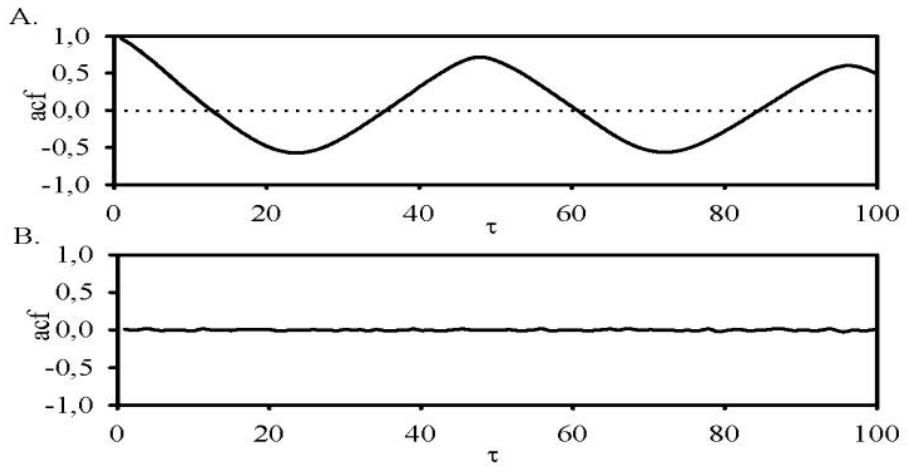

Figura 4. (A) autocorrelação da série de $\mathrm{CO}_{2}(35 \mathrm{~m})$ e $(B)$ de uma série de dados aleatórios.

Palú (2008) usou o método da Informação Mútua (ABARBANEL et al., 1993) para escolher o melhor tempo de defasagem temporal $(\tau)$ em análise de dados provenientes da torre micrometeorológica na Amazônia Legal em Sinop - MT, afirmando que um tempo de defasagem de 5 horas pode ser adotado em quaisquer que sejam as análises de séries temporais para a estimativa do parâmetro dimensão de correlação dos processos envolvidos na interação atmosfera-biosfera daquela floresta, independentemente de qual variável estiver sendo estudada e de que época do ano os dados se referem não existindo diferenciação entre as estações. Assim, para se calcular a função correlação (eq. 1 ), definiu-se para todas as variáveis uma defasagem temporal $(\tau)$ igual a $5 \mathrm{~h}$ $(\mathrm{m} \Delta \mathrm{t}=20 \times 15 \mathrm{~min}$, onde $\mathrm{m}$ é um número inteiro e $\Delta \mathrm{t}$ é um intervalo entre cada amostra), visto a baixa correlação entre as séries originais e as séries defasadas. Os resultados de Palú (2008) corroboram Baker \& Gollub (1996) que a escolha do parâmetro $\tau$ em situações práticas, pode ser regulada até que os resultados se tornem satisfatórios. Também que uma pequena variação no valor de $\tau$ não é significativa para alterar os resultados obtidos pelo método de análise.

\subsection{Atratores estranhos e dimensão fractal}

A dimensão fractal é uma medida comumente usada para medir a "estranheza" dos atratores, assim como o numero de graus de liberdade e informações estatísticas sobre o sistema. Neste artigo aplicamos o algoritmo de Grassberger \& Procaccia (1983) que é, dentre os diferentes procedimentos desenvolvidos para computar a dimensão fractal, o mais amplamente utilizado. A $D_{2}$ também provê o número de variáveis independentes necessária para descrever a evolução temporal da dinâmica do sistema com a dimensão de imersão $m$ (TAKENS, 1981) que tem como limite superior $2 D_{2}+1$ para modelar um sistema. Em um espaço de fase $m$-dimensional, a função correlação integrante $C(r)$ do atrator é dada por (GRASSBERGER \& PROCACCIA, 1983):

$$
C(r)=\frac{1}{n^{2}} \sum_{\substack{i, j=1 \\ i \neq j}}^{n} \theta\left(r-\left|\mathrm{X}_{\mathrm{i}}-\mathrm{X}_{j}\right|\right)
$$

em que $\theta$ é a função Heaviside, $\theta(x)=0$ se $x<0$, e $\theta(x)=1$ se $x>0$. 
A partir de uma pequena correlação $\varepsilon$ sondar-se a estrutura do Atrator. Se esta é uma linha, o número de pontos dentro de uma sondagem à distância $r$ de um ponto deve ser proporcional ao $r / \varepsilon$. Se for uma superfície este número deve ser proporcional a $(r / \varepsilon)^{2}$ e, de forma geral, se for uma dimensão $d$ deve ser proporcional a $(r / \varepsilon)^{d}$. Logo, para $r$ relativamente pequeno, $C(r)$ deverá variar conforme...

$$
C(r) \sim r^{d}
$$

Assim, como a dimensão de correlação $D_{2}$ do atrator é aproximadamente igual à dimensão fractal $d$, ela é dada pela declinação $\ln C(r)$ por $\ln r$ para um valor de $r$ infinitesimal crescente até a integração total do atrator. Ou seja, a dimensão fractal é obtida do prolongamento linear da figura "joelho" (Figura 5A e 5B) do qual são extraídos os coeficientes lineares (Figura 5C).

$$
\ln C(r)=d \ln r
$$

Afere-se a dimensionalidade mínima, $n$, do espaço de fase dentro do qual 0 atrator mencionado está imerso. $O$ parâmetro $n$ define o número mínimo de variáveis que devem ser consideradas na descrição da dinâmica do sistema, ou seja, o número mínimo de equações diferenciais de primeira ordem que podem conter as características qualitativas do sistema dinâmico estudado (GRASSBERGER \& PROCACCIA, 1983). Importante ressaltar que $D_{2}$ é necessariamente menor que $n$.
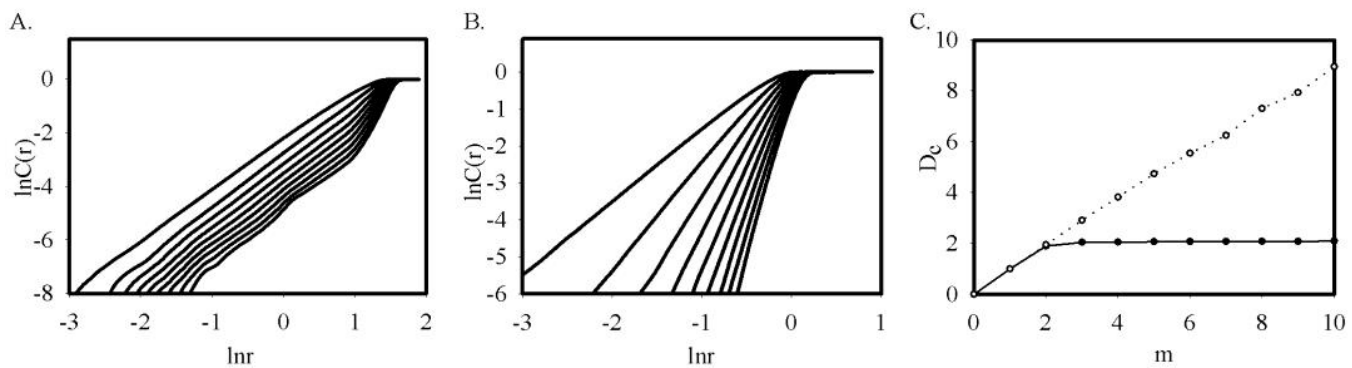

Figura 5. (a) $\ln C(r)$ vs $\ln r$ para valores crescentes de $m$ para o atrator reconstruído a partir da série $x$ de Lorenz e (b) $\ln C(r)$ vs $\ln r$ para uma série de dados aleatórios; e (c) saturação da dimensão de correlação versus dimensão de imersão $m$ para o atrator reconstruído a partir da série $\mathrm{x}$ de Lorenz com $D_{2} \approx 2,05 \pm 0,01$ e $n=3(\bullet)$ e a instauração para a série aleatória com dimensão infinita (o).

O algoritmo de Grassberger \& Procaccia (1983) consome um tempo de processamento excessivamente longo, aumentando significativamente esse tempo em análises de séries muito longas, pelo fato de exigir a quantificação e classificação das $N(N-1)$ distâncias (Eq. 1), onde $N$ é o número de vetores reconstruídos pela relação de Takens (1981). 


\section{RESULTADOS E DISCUSSÕES:}

\section{Dimensão de correlação $\mathrm{D}_{2}$}

Nesta seção são apresentados os resultados quanto à dimensão de correlação $\mathrm{D}_{2}$ associada a cada série temporal analisada. Com caráter ilustrativo, são apresentadas nas Figuras 6 até 11, para referenciar um mês de cada um dos períodos analisados. Assim são apresentados nestas figuras as curvas de $\operatorname{lnC}(r)$ versus Inr, a dimensionalidade " $d$ " quanto ao numero das variáveis do espaço de fase " $n$ ", e reconstrução tridimensional do atrator climático associado às séries temporais, respectivamente para as variáveis $\mathrm{Rn}$, $\mathrm{T}$ e UR, com um mês para cada período visto que esses meses serem representativos

Ainda quanto a estes resultados da dimensionalidade fractal dos atratores, são apresentados na Tabela 2 a dimensão de correlação $D_{2}$ média para todo cada período analisado e a classificação destes valores quanto às suas características, que podem ser periódicas, quase periódicas ou não periódicas (Tabela 3).

Tabela 2. Valores do parâmetro dimensão de correlação $\left(D_{2}\right)$ dos atratores reconstruídos a partir das variáveis $\mathrm{Rn}, \mathrm{T}$ e UR.

\begin{tabular}{|c|c|c|c|c|c|c|c|}
\hline \multicolumn{4}{|c|}{ Seca 2007} & \multicolumn{4}{|c|}{ Seca 2008} \\
\hline Mês/Variável & $\mathrm{Rn}$ & $\mathrm{T}$ & UR & Mês/Variável & $\mathrm{Rn}$ & $\mathrm{T}$ & UR \\
\hline Julho & 1,53 & 2,17 & 2,49 & Julho & 1,40 & 1,50 & 1,81 \\
\hline Agosto & 1,33 & 2,16 & 2,24 & Agosto & 1,50 & 1,70 & $2,35 *$ \\
\hline \multicolumn{4}{|c|}{ Chuva 2008} & \multicolumn{4}{|c|}{ Chuva 2009} \\
\hline Mês/Variável & $\mathrm{Rn}$ & $\mathrm{T}$ & UR & Mês/Variável & $\mathrm{Rn}$ & $\mathrm{T}$ & UR \\
\hline Janeiro & 1,91 & 2,30 & $2,30 *$ & Janeiro & 1,80 & 2,19 & 2,25 \\
\hline Fevereiro & 1,70 & 2,26 & 2,32 & Fevereiro & 1,80 & $2,44 *$ & $2,30 *$ \\
\hline \multicolumn{4}{|c|}{ Inundação 2008} & \multicolumn{4}{|c|}{ Inundação 2009} \\
\hline Mês/Variável & $\mathrm{Rn}$ & $\mathrm{T}$ & UR & Mês/Variável & $\mathrm{Rn}$ & $\mathrm{T}$ & UR \\
\hline Março & 1,47 & 2,00 & 1,54 & Março & 1,85 & 2,50 & 2,37 \\
\hline Abril & 1,64 & 2,35 & $2,35^{*}$ & Abril & 1,37 & 2,25 & 2,01 \\
\hline Maio & 1,60 & 1,95 & 1,90 & Maio & 1,53 & 1,52 & 2,07 \\
\hline
\end{tabular}

[*Valores não saturados no espaço de fase com 12 graus de liberdade $(n=12)$ ]

Os resultados de $D_{2(R n)}$ (dimensão de correlação para $R n$ ) saturou-se em todos os meses analisados mantendo-se entre $1<D_{2(R n)}<2$. Estes resultados possibilitam inferir que, dentre as séries temporais das variáveis analisadas e períodos considerados, $\mathrm{Rn}$ é a variável mais simples de ser modelada no Cambarazal do Pantanal Matogrossense. Houve variabilidade sazonal de Rn, 
com os maiores valores de $D_{2(R n)}$ no período chuvoso e os menores no período seco, o que está de acordo com a declinação solar anual, em que a média mínima Rn é em junho, e máxima é em dezembro (VAREJÃO, 2006; REICHARDT \& TIMM, 2004). A nebulosidade implica variabilidade no padrão da série temporal do $\mathrm{Rn}$, e consequentemente suas maiores dimensionalidades fractais no período chuvoso. O inverso se verifica no período seco, mesmo com as emissões de $\mathrm{CO}_{2}$ por queimadas, que afetam fortemente Rn (ARTAXO et al., 2005). 

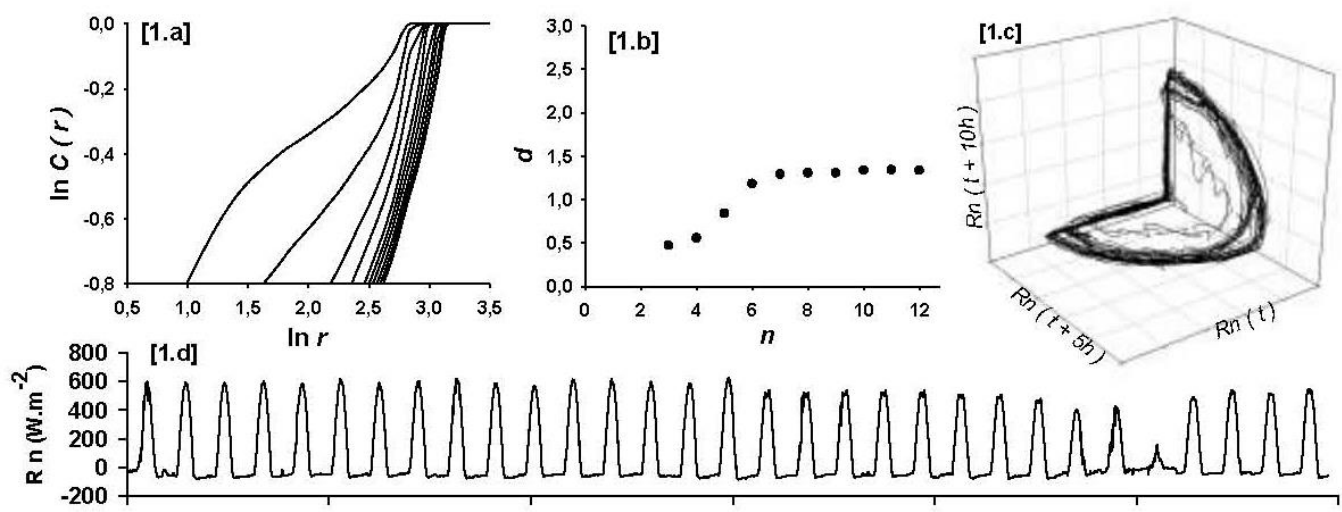

Agosto 2007
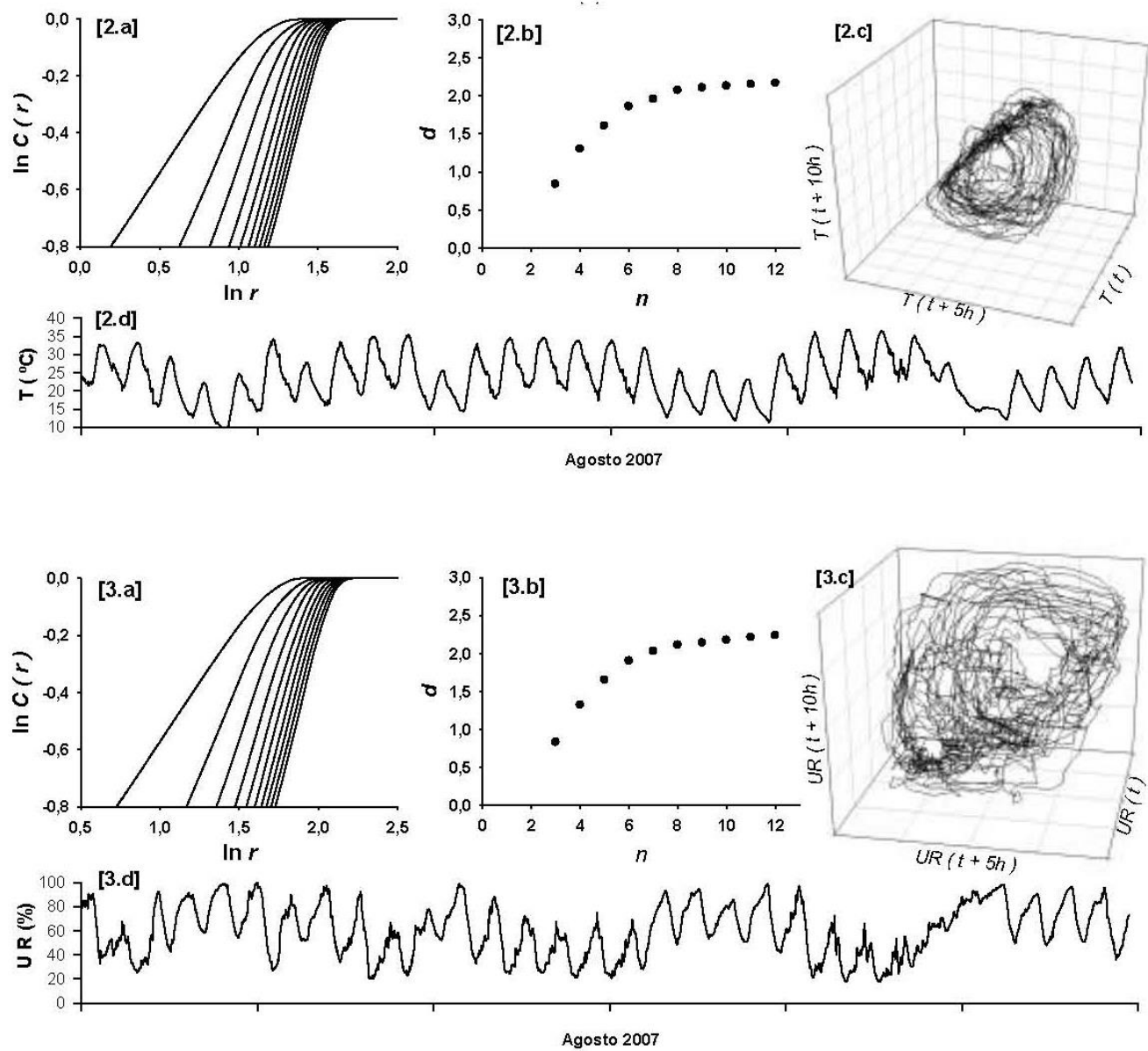

Figura 6. (a) InC(r) versus Inr, (b) dimensionalidade "d" quanto ao numero das variáveis do espaço de fase " $n$ ", (c) reconstrução do atrator climático, (d) série temporal, respectivamente para as variáveis (1) Rn, (2) T e (3) UR, período seco, agosto de 2007. 

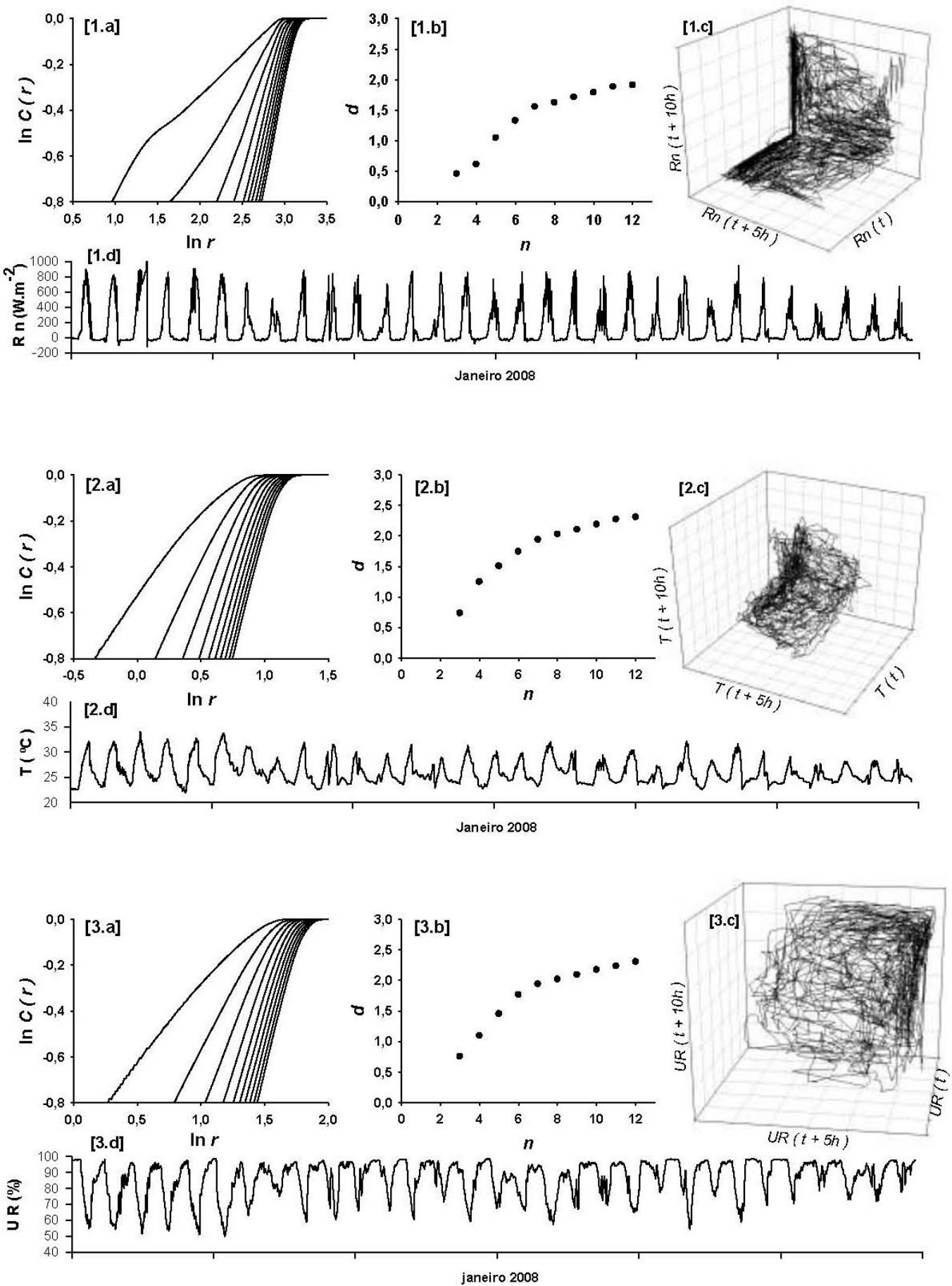

Figura 7. (a) $\ln C(r$ ) versus $\operatorname{lnr}$, (b) dimensionalidade " $d$ " quanto ao numero das variáveis do espaço de fase " $n$ ", (c) reconstrução do atrator climático, (d) série temporal, respectivamente para as variáveis (1) Rn, (2) T e (3) UR, período chuvoso, Janeiro 2008. 

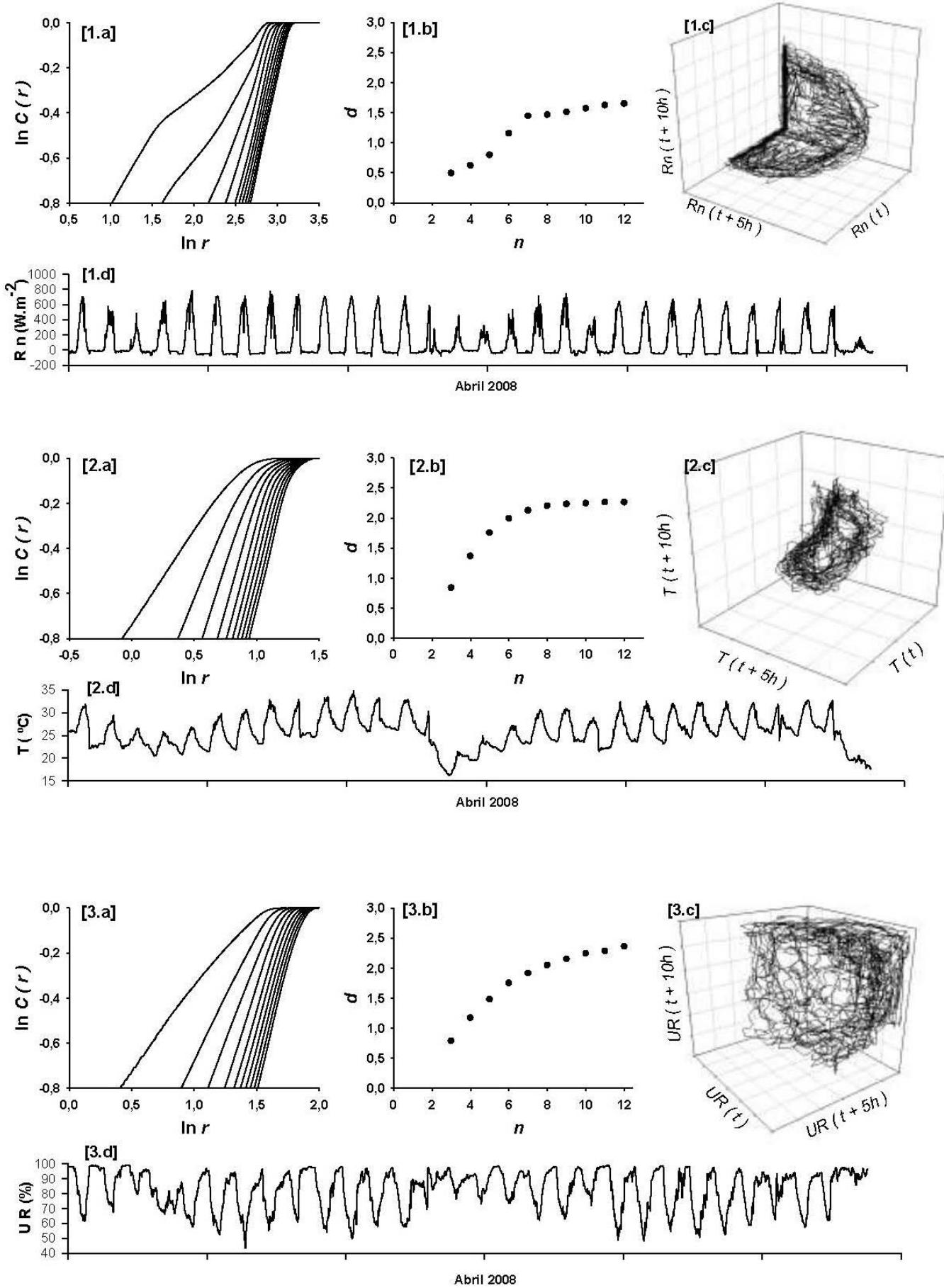

Figura 8. (a) $\operatorname{lnC}(r)$ versus $\ln r$, (b) dimensionalidade " $d$ " quanto ao numero das variáveis do espaço de fase " $n$ ", (c) reconstrução do atrator climático, (d) série temporal, respectivamente para as variáveis (1) Rn, (2) T e (3) UR, período Inundado, Abril de 2008. 

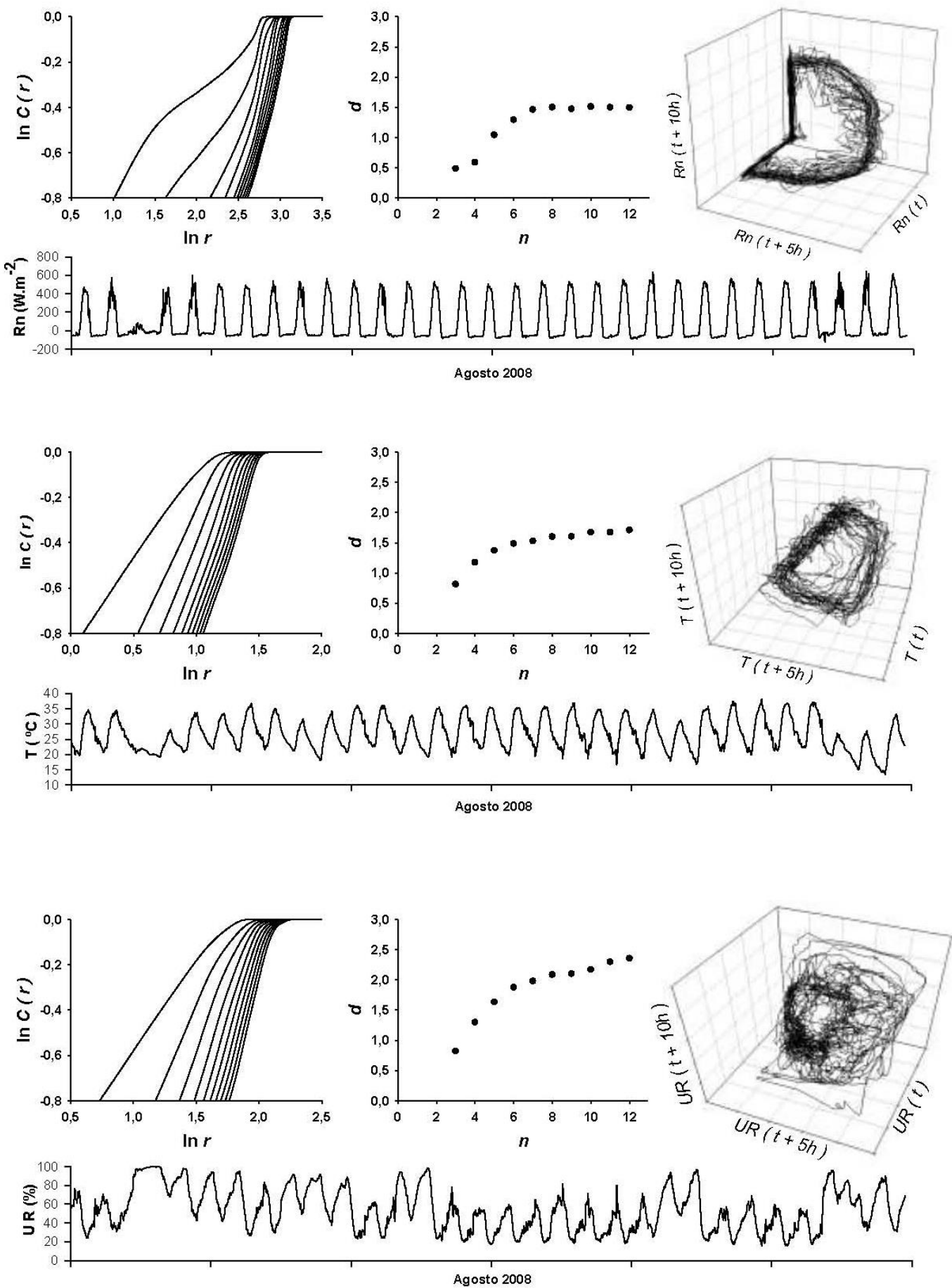

Figura 9. (a) $\ln C(r)$ versus $\ln r$, (b) dimensionalidade " $d$ " quanto ao numero das variáveis do espaço de fase " $n$ ", (c) reconstrução do atrator climático, (d) série temporal, respectivamente para as variáveis (1) Rn, (2) T e (3) UR, período Seco, Agosto de 2008. 

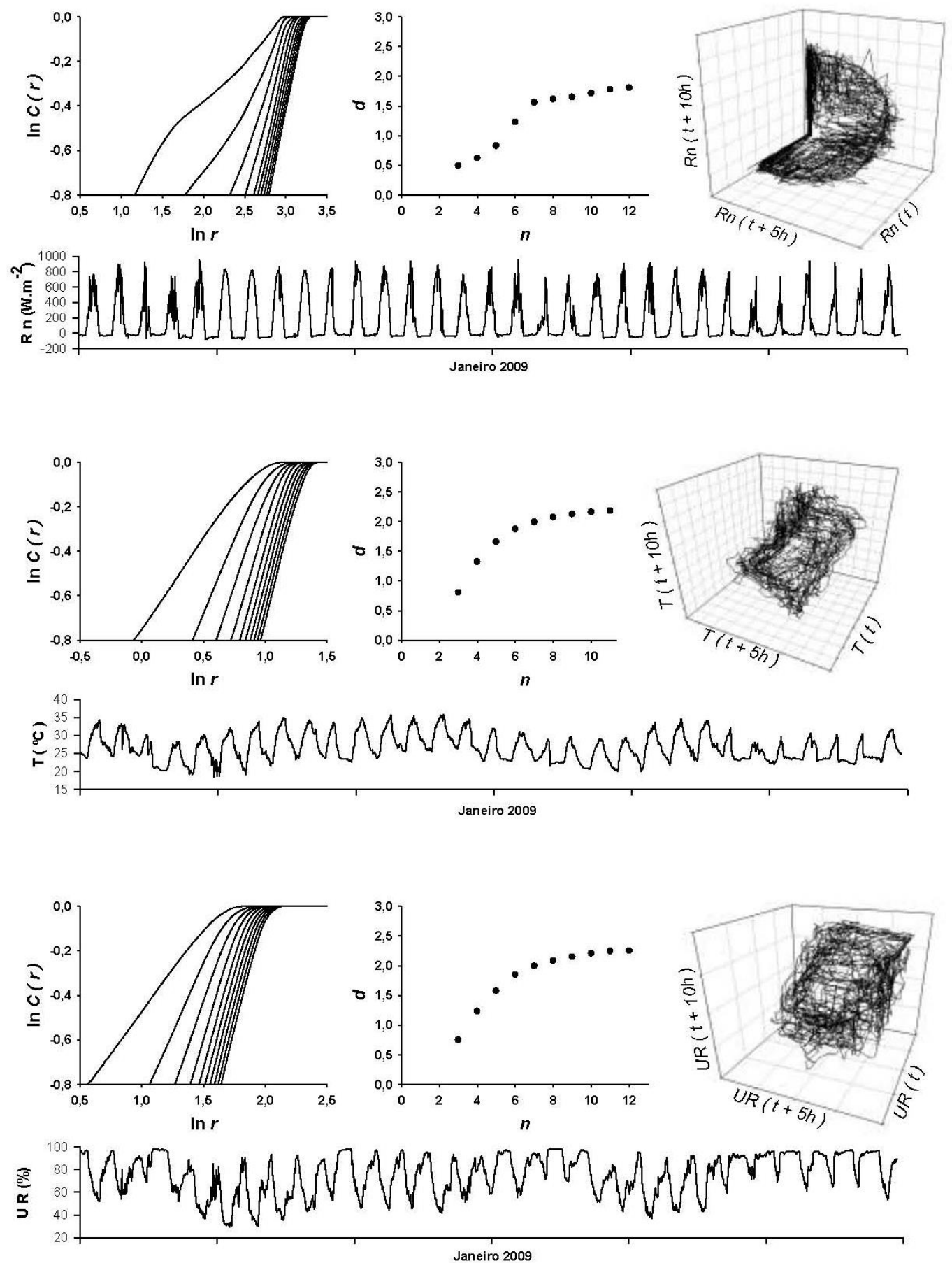

Figura 10. (a) $\ln C(r)$ versus $I n r$, (b) dimensionalidade " $d$ " quanto ao numero das variáveis do espaço de fase " $n$ ", (c) reconstrução do atrator climático, (d) série temporal, respectivamente para as variáveis (1) Rn, (2) T e (3) UR, período Chuvoso, Janeiro de 2008. 

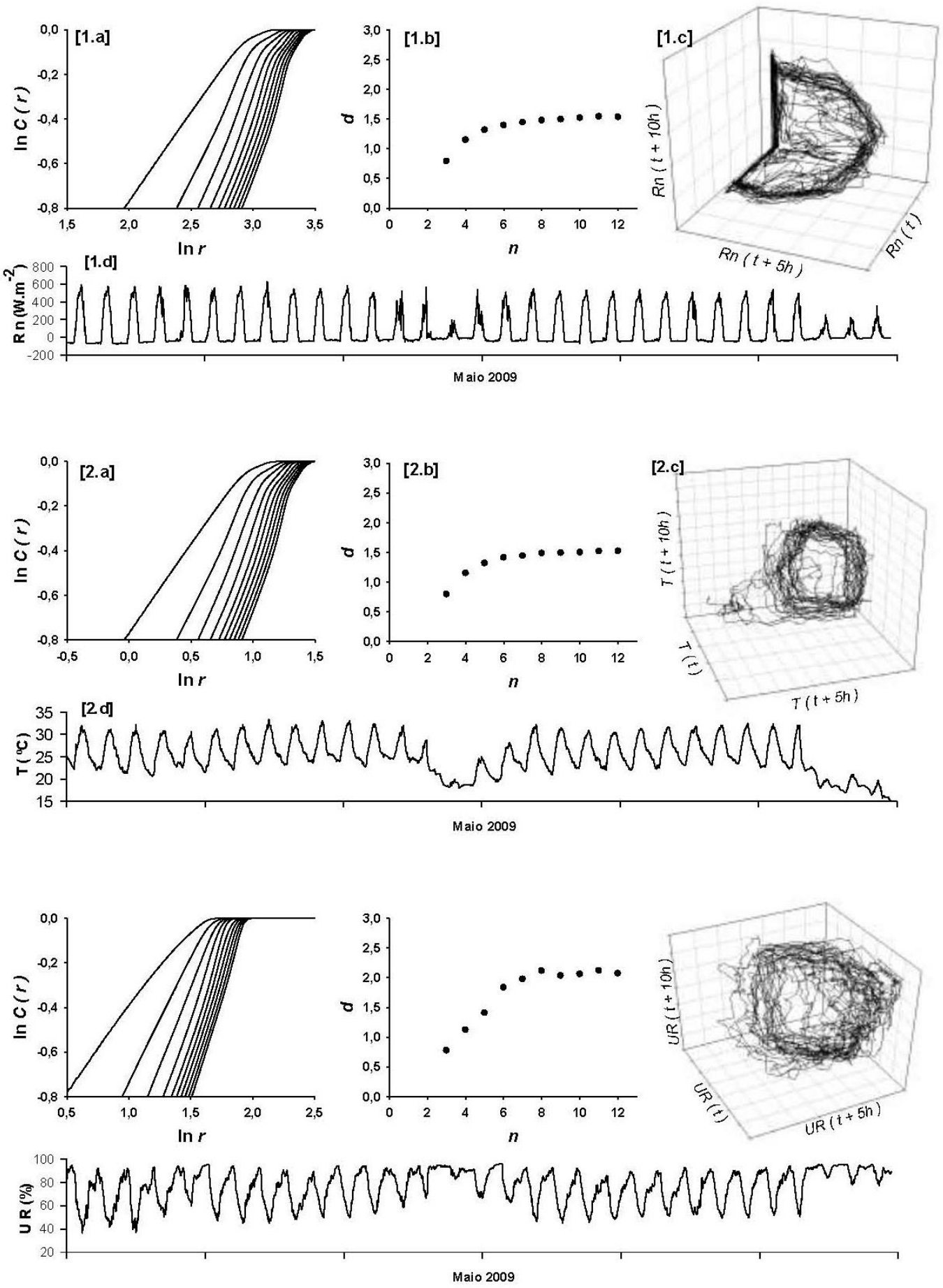

Figura 11. (a) $\ln C(r)$ versus $\operatorname{lnr}$, (b) dimensionalidade " $d$ " quanto ao numero das variáveis do espaço de fase " $n$ ", (c) reconstrução do atrator climático, (d) série temporal, respectivamente para as variáveis (1) Rn, (2) T e (3) UR, período Inundado, Maio de 2008. 
A série temporal da variável T apresentou um atrator com $D_{2(T)}>2$ no período chuvoso, não estabilizando em fevereiro de 2009. Os períodos inundados foram marcados por $D_{2(T)} \geq 2$ nos dois primeiros meses e $D_{2(T)}<2$ no terceiro mês. Ressalta-se que $T$ é um dos efeitos mais importantes do Rn, e que o aquecimento da atmosfera próxima à superfície terrestre ocorre principalmente por transporte de calor a partir do aquecimento da superfície pelos raios solares. As variações temporais e espaciais da temperatura do ar são condicionadas pelo balanço de energia na superfície. Assim, todos os fatores que afetam o balanço de energia na superfície influenciam também a temperatura do ar (PEREIRA et al., 2002).

Nas séries temporais de $\mathrm{Rn}$ observou-se picos próximos a 1000 W. $\mathrm{m}^{-2}$ no período chuvoso e picos próximos a $500 \mathrm{~W} \cdot \mathrm{m}^{-2}$ no período seco (MELLO, 2010). A variação sazonal da dimensionalidade (ou seja, diminuição de $D_{2(T)}$ ao final do período inundado) está estreitamente ligada ao balanço de energia do ecossistema (no que diz respeito ao aspecto astronômico de irradiação solar) ligados ao solstício e as estações do ano, manifestando-se nas diferentes médias de temperatura durante 0 ano.

No período seco de 2007, a $D_{2(T)}>2$, e no período seco de 2008 foi $D_{2(T)}<2$. A análise desses resultados relaciona-se com a dinâmica atmosférica global, que durante os meses de maio a setembro (como maior prevalência em julho e agosto) ocorre uma intensificação no mecanismo de produção de massas de ar frio nas imediações do polo Sul, deslocando-se no sentido sul-norte. Essas massas de ar frio, por vezes acompanhadas de nebulosidade, passam a atingir regiões de clima subtropical, tropical e, até mesmo equatorial, promovendo quedas bruscas na temperatura normalmente acompanhadas de ventos. Nessas ocasiões, regiões com medias anuais de temperatura extremamente elevadas podem apresentar subitamente quedas para patamares muito baixos, geralmente com duração de quatro a cinco dias. Em Cuiabá, a mínima pode atingir níveis inferiores a $5^{\circ} \mathrm{C}$ (BRASIL, 2003). A partir das séries temporais dos períodos secos analisados é possível observar que houve a chegada de frentes frias durante a seca 2007, e que esta promoveu uma oscilação da amplitude térmica diária (variando subitamente de entre $22^{\circ} \mathrm{C}$ e $32^{\circ} \mathrm{C}$ para aproximadamente $10^{\circ} \mathrm{C}$ e $15^{\circ} \mathrm{C}$ ), das temperaturas mínimas e máximas e da insolação durante alguns dias. Isto deslocou a trajetória dos estados do atrator no espaço de fase, o qual migrou de uma frequência periódica para amplitudes e frequências subjacentes. Infere-se que desprezando esses estados eventuais e de pouca duração do período seco de 2007 , o sistema apresentaria $D_{2(T)}$ muito próximo ao do período seco de 2008. Ressalta-se ainda que os valores observados para $D_{2(\mathrm{~T})}$ foram mais baixos do que os encontrados na literatura (SIVAKUMAR, 2004; 2009) demonstrando o caráter previsível da temperatura no Cambarazal.

As séries temporais de $T$ dos períodos chuvosos e parte dos períodos inundados, embora resultem em $D_{2(T)}>2$, caracterizaram-se por amplitude térmica menor 
do que o período seco, mesmo com mais energia disponível no sistema, devido aos valores maiores de UR considerando-se o alto calor específico da água assim como o efeito termorregulador do Cambarazal em função da sua biomassa (BIUDES et al., 2009).

A variável UR apresentou $D_{2(U R)}>2$ no período chuvoso, não se estabilizando em janeiro de 2008 e fevereiro de 2009. O período seco de 2007 apresentou $D_{2(\text { UR })}>2$, enquanto o período seco de 2008 oscilou do mês julho com $D_{2(\text { UR })}<2$ para agosto com $\mathrm{D}_{2(\mathrm{UR})}>2$ não estabilizado. O período inundado de 2008 configurou-se oscilante nos seus três meses, sendo $D_{2(\mathrm{UR})}<2, D_{2(\mathrm{UR})}>2$ não estabilizado e $D_{2(U R)}<2$. A inundação 2009 apresentou $D_{2(U R)} \geq 2$. Esta variável foi a que apresentou o maior número de insaturações, verificando-se em $66,67 \%$ dos períodos analisados, ou quatro períodos. Assim como amplamente conhecido pela comunidade científica, as séries temporais da variável UR apresentaram dependência inversa em relação à $T$, atingindo seus valores mínimos nas horas mais quentes do dia (PEREIRA et al., 2002). A amplitude dos valores de UR foi maior na seca (de 20 à $100 \%$ ) do que na chuva ( $50 \%$ à $100 \%$ ), mas os valores da $D_{2(\text { UR) }}$ tiveram pouca variação sazonalmente.

O ambiente do Pantanal Matogrossense parece apresentar atratores temporais (ou climáticos) não periódicos de baixa dimensionalidade com pouca variação sazonal, demonstrando estabilidade ecossistêmica. A baixa dimensionalidade caótica para o atrator da temperatura apresentou valores menores do que outras pesquisas (ZENG \& PIELKE, 1993).

A classificação quanto ao comportamento da variável Rn apresentou oscilação periódica autossustentável em todos os períodos analisados. O período chuvoso, com valores $D_{2(R n)}$ mais próximos de 2 caracterizou-se como tendência de oscilações periódicas autossustentáveis para oscilações quase-periódicas de duas frequências independentes.

O comportamento da variável T para o período chuvoso apresentou oscilações não periódicas e imprevisibilidade intrínseca. O mesmo ocorreu com o período seco de 2007. Já o período seco de 2008 apresentou oscilação periódica autossustentável. O período inundado foi marcado em 2008 por oscilações quase periódicas de duas frequências independentes e não periódicas, e 2009 por oscilações não periódicas tendendo a uma oscilação periódica autossustentável.

A variável UR apresentou oscilações não periódicas em todos os períodos, principalmente no período chuvoso, em que todas as classificações foram marcadamente não periódicas. No período seco, mas especificamente em agosto, observou-se comportamento não periódico para os dois anos analisados. Quanto ao período inundado, observou-se que maio 2008 e 2009 apresentaram oscilações quase periódicas de duas frequências independentes.

Ainda sobre as análises que não saturaram, observa-se que elas não são estocásticas, mas contem alguma componente caótica. Isto pode ser observado pela inclinação tendendo a saturação em $d$ versus $n$, e uma pequena variação enquanto $\mathrm{n}$ aumenta. 
A dimensão de imersão $n$, que representa número de equações diferencias necessárias para modelar a dinâmica da série temporal, apresentou os resultados contidos na Tabela 4.

Algumas curvas $D_{2}$ versus $n$ não saturaram totalmente, talvez isto seja devido a uma componente não linear acoplada à série temporal, ou seja, um baixo acoplamento com outros subsistemas de modo que haja influência secundária de outras variáveis.

O parâmetro $\mathrm{n}_{(\mathrm{Rn})}$ foi a mais bem comportado para todas as sazonalidades. Para o período seco está entre um intervalo de 6 a 7 graus de liberdade; no período inundado de 6 a 8 graus de liberdade, e no período chuvoso de 7 a 8 variáveis. 0 parâmetro $\mathrm{n}_{(\mathrm{T})}$ apresentou comportamento mais próximo do encontrado para o $\mathrm{n}_{(\mathrm{Rn})}$ no período seco e inundado, demonstrando maior acoplamento nestes períodos.

A $\mathrm{n}_{(\mathrm{UR})}$ apresentou os maiores números de graus de liberdade para o período seco. No período chuvoso oscilou entre 8 e 12* graus de liberdade, enquanto que na inundação manteve-se entre 7 e 12 graus de liberdade.

Tabela 3. Classificação das variáveis quanto ao comportamento: Periódico (I), Quase Periódico (II) e Não Periódico (III).

\begin{tabular}{|c|c|c|c|c|c|c|c|}
\hline \multicolumn{4}{|c|}{ Seca 2007} & \multicolumn{4}{|c|}{ Seca 2008} \\
\hline Mês/Variável & $\mathrm{Rn}$ & $T$ & UR & Mês/Variável & $\mathrm{Rn}$ & $T$ & UR \\
\hline Julho & I & III & III & Julho & I & I & I \\
\hline Agosto & I & III & III & Agosto & I & I & III \\
\hline \multicolumn{4}{|c|}{ Chuva 2008} & \multicolumn{4}{|c|}{ Chuva 2009} \\
\hline Mês/Variável & $\mathrm{Rn}$ & $T$ & UR & Mês/Variável & $\mathrm{Rn}$ & $T$ & UR \\
\hline Janeiro & I & III & III & Janeiro & I & III & III \\
\hline Fevereiro & I & III & III & Fevereiro & I & III & III \\
\hline \multicolumn{4}{|c|}{ Inundação 2008} & \multicolumn{4}{|c|}{ Inundação 2009} \\
\hline Mês/Variável & $\mathrm{Rn}$ & $\mathrm{T}$ & UR & Mês/Variável & $\mathrm{Rn}$ & $T$ & UR \\
\hline Março & I & II & I & Março & I & III & III \\
\hline Abril & I & III & III & Abril & I & III & II \\
\hline Maio & I & I & II & Maio & I & I & II \\
\hline
\end{tabular}


Tabela 4. Classificação da dimensão de imersão (n): número mínimo de graus de liberdade no espaço de fase das variáveis.

\begin{tabular}{|c|c|c|c|c|c|c|c|}
\hline \multicolumn{4}{|c|}{ Seca 2007} & \multicolumn{4}{|c|}{ Seca 2008} \\
\hline Mês/Variável & $\mathrm{Rn}$ & $T$ & UR & Mês/Variável & $\mathrm{Rn}$ & $\mathrm{T}$ & UR \\
\hline Julho & 7 & 8 & 11 & Julho & 6 & 7 & 7 \\
\hline Agosto & 7 & 8 & 8 & Agosto & 7 & 6 & $8^{*}$ \\
\hline \multicolumn{4}{|c|}{ Chuva 2008} & \multicolumn{4}{|c|}{ Chuva 2009} \\
\hline Mês/Variável & $\mathrm{Rn}$ & $\mathrm{T}$ & UR & Mês/Variável & $\mathrm{Rn}$ & $\mathrm{T}$ & UR \\
\hline Janeiro & 7 & 11 & $10 *$ & Janeiro & 7 & 8 & 8 \\
\hline Fevereiro & 7 & 9 & 8 & Fevereiro & 8 & $11 *$ & $12 *$ \\
\hline \multicolumn{4}{|c|}{ Inundação 2008} & \multicolumn{4}{|c|}{ Inundação 2009} \\
\hline Mês/Variável & $\mathrm{Rn}$ & $\mathrm{T}$ & UR & Mês/Variável & $\mathrm{Rn}$ & $\mathrm{T}$ & UR \\
\hline Março & 7 & 7 & 7 & Março & 8 & 11 & 10 \\
\hline Abril & 8 & 8 & $12 *$ & Abril & 7 & 8 & 7 \\
\hline Maio & 6 & 7 & 7 & Maio & 6 & 8 & 7 \\
\hline
\end{tabular}

* valores de $\mathrm{n}$ não saturados imersos em hiperespaço de fase de $12 \mathrm{n}$.

Todos os graus de liberdade do sistema ambiental estão interligados de alguma forma, logo temos fluxos de matéria, energia e momentum entre os diversos subsistemas que o compõem. O ecossistema Pantanal, objeto desta análise, é um subsistema do sistema global; ora se expande abarcando mais graus de liberdade ( $n$ ) e ora se contrai diminuindo o número das variáveis mais relevantes para compreensão dos estados dos atratores. Lorenz (1991) afirma "que o ambiente pode ser visto como subsistemas de baixo acoplamento. Neste caso, os estudos tentam avaliar a dimensão do subsistema."

Algumas curvas $D_{2}$ versus $n$ não saturaram totalmente, talvez isto seja devido a uma componente não linear acoplada à série temporal, ou seja, um baixo acoplamento com outros subsistemas de modo que haja influência secundária de outras variáveis.

O parâmetro $\mathrm{n}_{(\mathrm{Rn})}$ foi a mais bem comportado para todas as sazonalidades. Para o período seco está entre um intervalo de 6 a 7 graus de liberdade; no período inundado de 6 a 8 graus de liberdade, e no período chuvoso de 7 a 8 variáveis. O parâmetro $\mathrm{n}_{(\mathrm{T})}$ apresentou comportamento mais próximo do encontrado para o $\mathrm{n}_{(\mathrm{Rn})}$ no período seco e inundado, demonstrando maior acoplamento nestes períodos.

A $\mathrm{n}_{(\mathrm{UR})}$ apresentou os maiores números de graus de liberdade para o período seco. No período chuvoso oscilou entre 8 e $12 *$ graus de liberdade, enquanto que na inundação manteve-se entre 7 e 12 graus de liberdade. 
Todos os graus de liberdade do sistema ambiental estão interligados de alguma forma, logo temos fluxos de matéria, energia e momentum entre os diversos subsistemas que o compõem. O ecossistema Pantanal, objeto desta análise, é um subsistema do sistema global; ora se expande abarcando mais graus de liberdade $(n)$ e ora se contrai diminuindo o número das variáveis mais relevantes para compreensão dos estados dos atratores. Lorenz (1991) afirma "que o ambiente pode ser visto como subsistemas de baixo acoplamento. Neste caso, os estudos tentam avaliar a dimensão do subsistema."

\section{Sazonalidade em relação aos parâmetros $D_{2}$ e $n$}

Em média, o parâmetro $D_{2}$ das variáveis analisadas apresentou uma variação entre os períodos secos, chuvosos e inundados. Este comportamento sistemático da dimensionali ${ }_{d}$ ade dos atratores pode ser observado pelas médias dos períodos plotados na figura 12 .
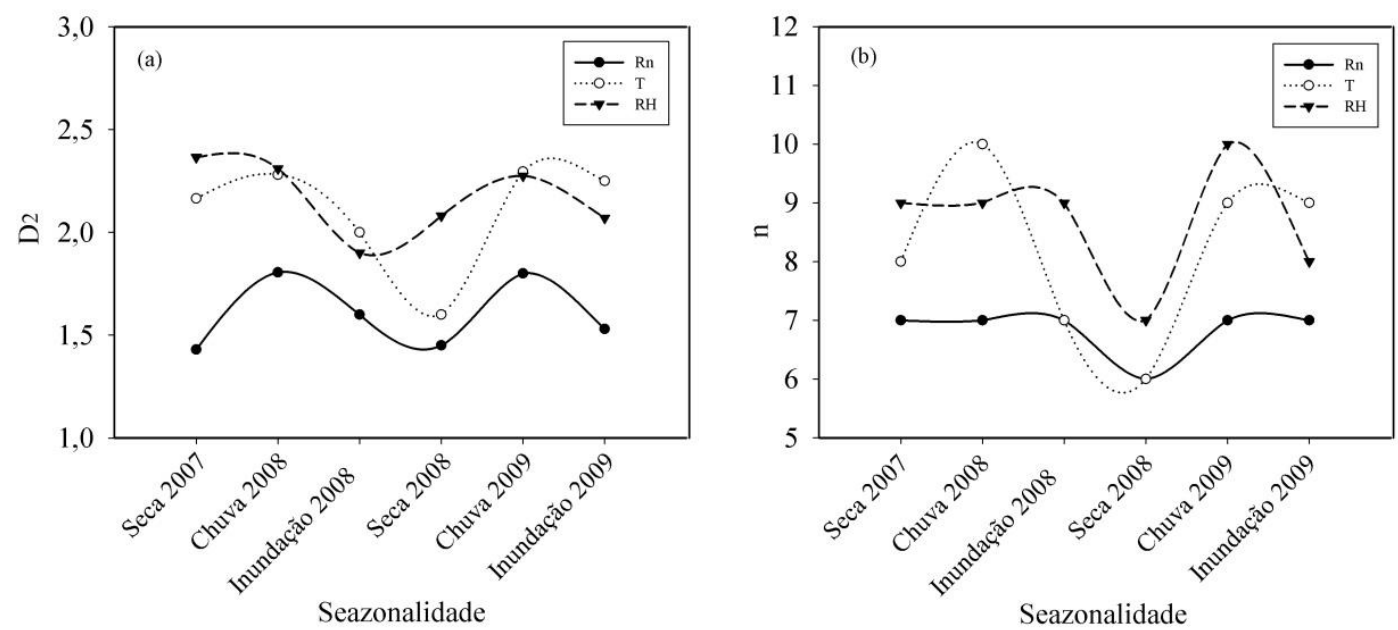

Figura 12. Tendências sazonais dos parâmetros (a) $D_{2}$ e (b) $n$.

O Rn e T apresentaram o mesmo comportamento, em média, quanto a variação da $D_{2}$ sazonalmente, aumentando no período chuvoso e diminuindo no período seco. Para essas variáveis, o período de inundação se apresenta como transição chuva-seca.

A UR apresentou, em média, seus menores valores $D_{2}$ no período de inundação, visto a Floresta de Cambarazal/Pantanal ser monodominante, estar alagada com aproximadamente um metro de lâmina d'água e contar com pouca ocorrência de chuvas. Nos períodos secos e chuvosos foram encontradas as maiores dimensionalidades, vindo em seguida os períodos inundados.

As variáveis Balanço de Radiação (Rn), Temperatura do $\operatorname{Ar}(T)$ e Umidade Relativa do $\operatorname{Ar}$ (UR) apresentaram uma variação sazonal quanto ao número de graus de liberdade. Todas as séries temporais das variáveis analisadas diminuíram, em média, o número de graus de liberdade no período seco. Isto 
implica em uma maior possibilidade de entendimento dessas variáveis no período seco.

O Saldo de Radiação (Rn) apresentou uma diminuição de aproximadamente 1 (um) grau de liberdade no período seco, já as variáveis Temperatura do $\operatorname{Ar}(\mathrm{T})$ e Umidade Relativa do Ar (UR) diminuíram de 3 a 4 graus de liberdade. O período inundado apresentou-se como transição chuva-seca.

Desta maneira, as variações sazonais da dimensão de correlação $D_{2}$ implica em um comportamento mais errático das variáveis no período chuvoso, o que possivelmente vem a dificultar um processo de modelagem. E ainda, as variações sazonais da dimensão de imersão $n$, implicam em um maior número de variáveis necessárias para essa modelagem nos períodos chuvosos.

Assim como Hastings et al. (1993) e McCann (2000), especula-se sobre a possibilidade da existência de relação diretamente proporcional entre a complexidade de um ecossistema e a sua estabilidade dinâmica.

\section{CONCLUSÃO}

Séries temporais de variáveis micrometeorológicas medidas em floresta de Cambarazal, ecossistema Pantanal Matogrossense apresentaram atratores estranhos de baixa dimensionalidade sendo que das variáveis analisadas 0 atrator reconstruído da UR se exibe como o mais complexo de ser modelada, e do Rn o mais simples.

Os parâmetros $D_{2}$ e $n$ oscilaram em fase sazonalmente com picos nos períodos chuvosos para todas as variáveis estudas.

Das relações sazonais quanto ao parâmetro $n$, o Rn apresentou uma diminuição de aproximadamente 1 (um) grau de liberdade do período chuvoso para o período seco, já as variáveis Temperatura do $\operatorname{Ar}(\mathrm{T})$ e Umidade Relativa do $\mathrm{Ar}$ (UR) diminuíram de 3 a 4 graus de liberdade na transição chuva-seca. O período inundado apresentou-se como transição chuva-seca.

Sugere-se que a Floresta de Cambarazal no Pantanal aja como regulador da periodicidade da Umidade Relativa do $\operatorname{Ar}$ (UR), o que pode ser mais bem observado no período seco em que as chuvas menos frequentes permitem a aferição e análise dos valores de UR mais ligados à evapotranspiração.

\section{AGRADECIMENTOS}

À Coordenação de Aperfeiçoamento de Pessoal de Nível Superior (CAPES) pelo apoio com bolsa de estudos de Pós Graduação, e à Fundação de Amparo à Pesquisa do Estado de Mato (FAPEMAT) através do Programa de Apoio a Núcleos de Excelência (PRONEX) pelo apoio financeiro ao projeto de pesquisa processo n. $823971 / 2009$. 


\section{BIBLIOGRAFIA}

ABARBANEL, H. D. I.; BROW, R.; SIDOROWICH, J. J.; TSIMRING, L. S. The analysis of observed chaotic data in physical systems. Reviews of Modern Physics, vol. 65, no 4, p. 1331-1392, 1993.

ARIEIRA, J.; NUNES DA CUNHA, C. Fitossociologia de uma floresta inundável monodominante de Vochysia divergens Pohl (Vochysiaceae), no Pantanal Norte, MT, Brasil. Acta Botanica Brasilica, vol. 20, p. 569-580, 2006.

ARTAXO, P.; GATTI, L. V.; LEAL, A. M. C.; LONGO, K. M.; FREITAS, S. R.; LARA L. L.; PAULIQUEVIS, T. M.; PROCÓPIO, A. S.; RIZZO, L. V. Química atmosférica na Amazônia: a floresta e as emissões de queimadas controlando a composição da atmosfera amazônica. Acta Amazônica, vol. 35, no 2, p. 185-196, 2005.

BAKER, G. L.; GOLLUB, J. P. Chaotic dynamics: an introduction. New York, USA: Cambridge University Press; 1996. 256p.

BELLAVER, V. Difusividade térmica do solo em área monodominante de cambará no norte do Pantanal Matogrossense. 2010. 54p. Dissertação (Mestrado em Física Ambiental) - Instituto de Física, Universidade Federal de Mato Grosso, Cuiabá.

BIUDES, M. S.; CAMPELO JÚNIOR, J. S.; NOGUEIRA, J. S.; SANCHES, L. Estimativa do balanço de energia em cambarazal e pastagem no norte do pantanal pelo método da razão de Bowen. Revista Brasileira de Meteorologia, 24, no 2, p. 135-143, 2009.

BRASIL. Ministério da Integração Nacional. Secretaria Nacional de Defesa Civil. Manual de desastres - volume I - desastres naturais. Brasília; 2003.

CAMPANHARO, A. S. L. O.; RAMOS, F. M.; MACAU, E. E. N.; ROSA, R. R.; BOLZAN, M. J. A.; SÁ, L. D. A. Searching chaos and coherent structures in the atmospheric turbulence above the Amazon forest. Philosophical Transactions of The Royal Society A, vol. 366, p. 579-589, 2008.

CAMPELO JÚNIOR, J. H.; PRIANTE FILHO, N.; CASEIRO, F.T. Caracterização macroclimática de Cuiabá. In: Anais do III Encontro Nacional de Estudos Sobre o Meio Ambiente. Londrina, 1991. p. 545-552.

COSTA, M. H.; PIRES, G. F. Effects of Amazon and Central Brazil deforestation scenarios on the duration of the dry season in the arc of deforestation. International Journal of Climatology, vol. 30, p. 1970-1979, 2010.

FRAEDRICH K. Estimating the dimensions of weather and climatic attractors. Journal of the Atmospheric Sciences, vol. 43, p. 419-432, 1986.

GALLEGO, M. C.; GARCIA, J.A.; CANCILLO, M. L. Characterization of atmospheric turbulence by dynamical systems techniques. Boundary-Layer Meteorology, vol. 100, p. 375-392, 2001.

GRASSBERGER, P.; PROCACCIA, I. Characterization of strange attractors. Physical Review Letters, vol. 50, no 5, p. 346-349, 1983.

HASENACK, H.; CORDEIRO, J. L. P.; HOFMANN, G. S. O Clima da RPPN SESCPantanal. Porto Alegre, Brasil: UFRGS; 2003.

HASTINGS, A.; HOM, C. L.; ELLNER, S.; TURCHIN, P.; GODFRAY, H. C. J. Chaos in ecology: is mother nature a strange attractor? Annual Review of Ecological Systems, vol. 24 p. 1-33, 1993.

JUNK, W. J. Long-term environmental trends and the future of tropical wetlands. Environmental Conservation, vol. 29, no 4, p. 414-435, 2002.

JUNK, W. J.; NUNES DA CUNHA, C. Pantanal: a large South American wetland at a crossroads. Ecological Engineering Elsevier, vol. 24: p. 391-401, 2005.

LEWIS, S. L.; BRANDO, P.M.; PHILLIPS, O. L.; VAN DER HEIJDEN, G. M. F.; NEPSTAD, D. The 2010 Amazon Drought. Science, vol. 331, p. 554, 2011.

LORENZ, E. N. Deterministic nonperiodic flow. Journal of Atmospheric Sciences, vol. 20, p. 130-141, 1963.

LORENZ, E. N. Dimension of weather and climate attractors. Nature, vol. 353, p. 241244, 1991.

McCANN, K. V. The diversity-stability debate. Nature, vol. 405: p. 228-233, 2000.

MELLO, G. J. Análise de Séries Temporais de Variáveis Micrometeorológicas Medidas em Floresta de Cambarazal no Pantanal Matogrossense Utilizando a 
Teoria da Complexidade. 2010. 78p. Dissertação (Mestrado em Física Ambiental) Instituto de Física, Universidade Federal de Mato Grosso, Cuiabá.

NICOLIS, C.; NICOLIS, G. Is there a climatic attractor? Nature 1984, 311: 529-32.

NICOLIS, G.; PRIGOGINE, I. Exploring complexity: an introduction. New York, USA: W. H. Freeman and Company; 1998. 313p.

NOBRE, C. A.; FISCH, G.; ROCHA, H. R.; LYRA, R. F. F.; ROCHA, E. P.; COSTA, A. C. L.; UBARANA, V. N. Observations of the atmospheric boundary layer in Rondônia. In: GASH, J. H. C.; NOBRE, C. A.; ROBERTS, J. M.; VICTORIA, R. L. (Eds.) Amazonian Deforestation and Climate. JM Wiley and Sons, 1996. p. 413-424.

PALÚ, A. E. R. Determinação do tempo de defasagem mais adequado para análise de séries temporais de variáveis microclimatológicas medidas numa floresta de transição no norte de Mato Grosso. 2008. 38p. Dissertação (Mestrado em Física Ambiental) - Instituto de Física, Universidade Federal de Mato Grosso, Cuiabá.

PEITGEN, H.; JORGENS, H.; SAUPE, D. Chaos and fractals: new frontiers of science. New York, USA: Springer-Verlag, 2004. 864p.

PEREIRA, R. A.; ANGELOCCI, L. R.; SENTELHAS, P. C. Agrometeorologia: fundamentos e aplicações práticas. Guaíba, Brasil: Agropecuária; 2002. 478p.

POVEDO-JARAMILLO, G.; PUENTE, C. E. Strange attractors in atmospheric boundarylayer turbulence. Boundary-Layer Meteorology, vol. 64, p. 175-197, 1993.

REICHARDT, K.; TIMM, L. C. Solo, planta e atmosfera: conceitos, processos e aplicações. Barueri, Brasil: Manole Ltda; 2004. 478p.

RUELLE, D.; TAKENS, $F$. On the nature of turbulence. Communications in Mathematical Physics, vol. 20, p. 167-192, 1971.

SIVAKUMAR B. Nonlinear dynamics and chaos in hydrologic systems: latest developments and a look forward. Stochastic Environmental Research and Risk Assessment, vol. 23, p. 1027-1036, 2009.

SIVAKUMAR, B. Chaos theory in geophysics: past, present and future. Chaos Solitons \& Fractals Elsevier, vol. 19, no 2, p. 441-462, 2004.

TAKENS, F. Detecting strange attractors in turbulence. In: RAND, D. A.; YOUNG, L. S.; editors. Dynamical Systems and Turbulence, Lecture Notes in Mathematics, vol. 898. Berlin: Springer-Verlag; 1981. pp. 366-381.

VAREJÃO-SILVA, M. A. Meteorologia e climatologia. Brasília, Brasil: INMET; 2006.

WALDROP, M. M. Complexity, the emerging science at the edge of order and chaos. New York, USA: Touchstone; 1992. 380p.

XIN, L.; FEI, H.; GANG, L. Characteristics of chaotic attractors in atmospheric boundary layer turbulence. Boundary-Layer Meteorology, vol. 99, p. 335-345, 2001.

ZENG, X.; PIELKE, R. A. What does a low-dimensional weather attractor mean? Physics Letter A, vol. 175, p. 299-304, 1993.

ZENG, X.; PIELKE, R. A.; EYKHOLT, R. Estimating the fractal dimension and the predictability of the atmosphere. Journal of the Atmospheric Sciences, vol. 48, p. 649-659, 1992. 